\title{
Ferulic acid combined with astragaloside IV protects against vascular endothelial dysfunction in diabetic rats
}

\author{
Yonghui Yin ${ }^{1}$, Fanghua $\mathrm{Qi}^{2}$, Zhenhua Song ${ }^{3}$, Bo Zhang ${ }^{4}$, Jialin Teng ${ }^{3, *}$ \\ ${ }^{1}$ Department of Endocrinology, the First Affiliated Hospital of Shandong University of Traditional Chinese Medicine, Ji'nan, \\ Shandong, China; \\ ${ }^{2}$ Department of Traditional Chinese Medicine, Shandong Provincial Hospital affiliated to Shandong University, Ji'nan, \\ Shandong, China; \\ ${ }^{3}$ Shandong University of Traditional Chinese Medicine, Ji'nan, Shandong, China; \\ ${ }^{4}$ Department of Traditional Chinese Medicine and Traumatology, the Affiliated Hospital of Shandong Academy of Medical \\ Sciences, Ji'nan, Shandong, China.
}

\begin{abstract}
Summary Dysfunction of the endothelium is regarded as an important factor in the pathogenesis of vascular disease in diabetes mellitus (DM). Unfortunately, prevention of the progression of vascular complications of DM remains pessimistic. Ferulic acid and astragaloside IV, isolated from traditional Chinese medicine Angelica sinensis and Radix astragali respectively, exhibit potential cardio-protective and anti-hyperglycemic properties. In the present study, we investigated the protective effects and underlying mechanism of ferulic acid and astragaloside IV against vascular endothelial dysfunction in diabetic rats. After the diabetic rat model was established using streptozotocin, sixty rats were divided into 6 groups (control, model, ferulic acid, astragaloside IV, ferulic acid + astragaloside IV, and metformin) and treated for $\mathbf{1 0}$ weeks. Blood samples were collected to measure levels of hemoglobin A1c (HbAlc), triglyceride (TG), total cholesterol (TC), low density lipoprotein cholesterol (LDL-C), low density lipoproteins (Ox-LDL), alanine aminotransferase (ALT), aspartate aminotransferase (AST) and creatinine (Cr), nitric oxide (NO) and endothelial nitric oxide synthase (eNOS), and abdominal aorta tissue samples were collected for observing histological morphology changes of endothelium and detecting gene and protein

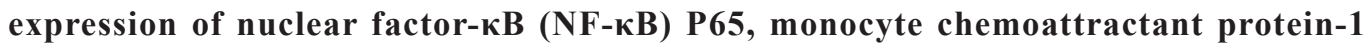
(MCP-1), and tumor necrosis factor $\alpha$ (TNF- $\alpha)$. We found that ferulic acid combined with astragaloside IV was capable of improving the structure of the aortic endothelium wall, attenuating the increase of HbAlc, TG, TC, LDL-C and Ox-LDL, promoting the release of NO and eNOS, and inhibiting over-activation of MCP-1, TNF- $\alpha$, and NFP65, without damage to liver and kidney function. In conclusion, ferulic acid combined with astragaloside IV exhibited significant protective effects against vascular endothelial dysfunction in diabetic rats through the NF-KB pathway involving decrease of Ox-LDL, increase of NO and eNOS, and activation of NF-KB P65, MCP-1 and TNF- $\alpha$.
\end{abstract}

Keywords: Ferulic acid, astragaloside IV, vascular endothelial dysfunction, diabetes mellitus

\section{Introduction}

Diabetes mellitus (DM) is a serious and complex chronic disease, which is dramatically increasing throughout the

\footnotetext{
*Address correspondence to:

Dr. Jialin Teng, Shandong University of Traditional Chinese Medicine, Ji'nan, Shandong, China.

E-mail:sd.jia@163.com
}

world. It has reached epidemic proportions worldwide and is associated with a large economic burden. According to a recent report, the number of diabetics is estimated to reach 439 million by 2030 worldwide (1). Diabetics show several complications, especially vascular complications (e.g., coronary insufficiency, cerebrovascular, and peripheral vascular disease), which are the main etiology for a great percentage of morbidity and mortality (2). Patients with DM exhibit complex 
vascular changes, such as an accelerated atherosclerosis process and hypercoagulability (3). Atherosclerosis is a major factor that accelerates diabetic vascular complications and vascular endothelial dysfunction is considered to be the earliest detectable abnormality in the process of atherosclerosis (4). Thus, improving vascular endothelial dysfunction plays an important role in DM treatment.

It is well-known that hyperglycemia-induced oxidative stress and inflammation play an important role in development of vascular endothelial dysfunction (5). Hyperglycemia stimulates the body to produce a large number of oxygen free radicals and then induces oxidative stress which damages vascular endothelial cells and causes DM vascular complications (6). Low density lipoproteins (Ox-LDL), an important product of oxidative stress, after binding with a specific receptor (Lectin-like OX-LDL receptor-1, LOX-1) on the surface of vascular endothelial cells, activates nuclear factor- $\mathrm{KB}$ (NF-kB), and mediates expression of some cytokines or inflammatory molecules including tumor necrosis factor $\alpha$ (TNF- $\alpha$ ), intercellular adhesion molecule-1 (ICAM-1), vascular cell adhesion molecule-1 (VCAM-1), as well as monocyte chemoattractant protein-1 (MCP-1) $(7,8)$. These cytokines facilitate the adhesion of neutrophils and monocytes to endothelium affecting production of endothelium-derived nitric oxide (NO), impairing endothelial dependent vasodilation function, inducing inflammatory damage of vascular walls, and initiating atherosclerosis (9).

Although drugs and changing life styles have been widely promoted to control the complications of DM, unfortunately, the prevention of the progression of vascular complications in diabetic patients remains pessimistic (3). Therefore, an effective approach to prevent or delay the development and progression of diabetic vascular complications is urgently needed. In recent years, application of traditional Chinese medicines (TCMs) for DM and its related complications has received increasing attention due to their wide availability, low side effects, and proven therapeutic mechanisms and benefits. Many TCMs and their active components have been reported to have antihyperglycemic effects, for example Angelica sinensis, Radix astragali, Radix rehmanniae, Panax ginseng $(10,11)$.

Angelica sinensis, which is called Dang-Gui in Chinese, has been mostly used to treat gynecological conditions and anemia for more than two thousand years in East Asia (12). In recent studies, Angelica sinensis and its active components have been shown to have multiple properties including cardio-protective, immune-modulatory, anti-oxidative, anti-inflammatory and so on (13). Ferulic acid (Figure 1A), as one of the major active components of Angelica sinensis, can effectively improve endothelial function in patients with coronary heart disease (14). Radix astragali, which is

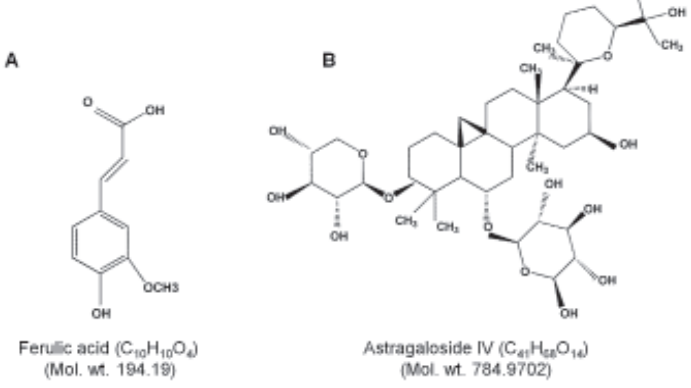

Figure 1. The structure of ferulic acid (A) and astragaloside IV (B).

known as Huang-Qi in Chinese, is traditionally used for treatment of diabetes, cardiovascular diseases, and wound healing, exerting anti-hyperglycemic, antioxidant, anti-inflammatory and immune-modulatory effects $(15,16)$. Recently, some active components isolated from Radix astragali including astragaloside IV (Figure 1B) have been demonstrated to have effects of alleviating glucose intolerance, insulin resistance, and hypertriglyceridemia in DM (16,17). Angelica sinensis combined with Radix astragali composes Dang-Gui-BuXue-Tang which is a classical prescription in traditional Chinese medicine for treating inflammation and ischemic diseases (18). Moreover, Dang-Gui-Bu-Xue-Tang is proposed as a potentially useful adjuvant therapy for patients with insulin resistance and/or patients who wish to increase insulin sensitivity (19). In a word, Angelica sinensis and Radix astragali either used alone or in combination possess effective cardio-protective and antihyperglycemic activities. We speculate that ferulic acid combined with astragaloside IV should exhibit effects against vascular complications of DM.

Thus, in the present study, we investigated the protective effects of ferulic acid and astragaloside IV against vascular endothelial dysfunction in diabetic rats. Furthermore, the underlying mechanisms for ferulic acid- and astragaloside IV-induced protective effects were investigated.

\section{Materials and Methods}

\subsection{Chemicals}

Ferulic acid and astragaloside IV were purchased from Chengdu Kang Bang Biological Technology Co., Ltd., Chengdu, China. Metformin was purchased from Shanghai Squibb pharmaceutical Co., Ltd., Shanghai, China. They were diluted in $55^{\circ} \mathrm{C}$ normal saline immediately before each experiment.

\subsection{Reagents}

High-fat and high-sugar diets were purchased from Shanghai Slac Laboratory Animal Co., Ltd., Shanghai, China. Streptozotocin was purchased from Sigma, St. 
Louis, MO, USA. Hemoglobin A1c (HbAlc) detection kit, NO kit and endothelial nitric oxide synthase (eNOS) kits were purchased from Nanjing Jiancheng Bioengineering Institute, Nanjing, China. The antiTNF- $\alpha$, anti-MCP-1, anti- NF- $\mathrm{B}$ P65, anti- $\beta$-actin antibodies and second antibodies were purchased from Santa Cruz Biotechnology (Santa Cruz, CA, USA). Diaminobenzidine (DAB) horseradish peroxidase color development kit was purchased from Beyotime Institute of Biotechnology (Shanghai, China). Ox-LDL, TNF- $\alpha$, and MCP-1 enzyme-linked immune-sorbent assay (ELISA) kits were purchased from Shanghai Dingguo Technology Co., Ltd., Shanghai, China. TRIzol reagent and SuperScript II First-Strand Synthesis System were purchased from Invitrogen (Grand Island, NY, USA). SYBR Premix Ex TaqTM kit was purchased from TaKaRa Bio Inc., Dalian, China.

\subsection{Diabetes rat model and drug administrations}

Male Wistar rats (Animal experimental center of Shandong University, Ji'nan, China) weighting approximately $180-220 \mathrm{~g}$ were used in this study. They were kept in a room with a $12 \mathrm{~h} / 12 \mathrm{~h}$ light/dark cycle and appropriate temperature $\left(22-26^{\circ} \mathrm{C}\right)$ and humidity (55\%), and given food and water ad libitum. After adaptive feeding for one week with blood glucose measured in the normal range, 60 rats were randomly divided into control group $(n=10)$ and model group $(n=50)$. The rats in the control group were fed normal chow diets, while the other rats were fed high-fat and high-sugar diets (containing 10.00\% lard, 20.00\% sucrose, $10.0 \%$ yolk powder, $0.50 \%$ sodium cholate and $59.50 \%$ normal chow) for 6 weeks. After an overnight fast, the rats in the model group were intraperitoneally injected with streptozotocin $(40 \mathrm{mg} / \mathrm{kg})$ and the rats in the control group were intraperitoneally injected with vehicle citrate buffer at a dose of $1 \mathrm{~mL} / \mathrm{kg}$. Seventy two hours after injection, tail blood samples were taken to test random blood glucose. Rats with random blood glucose levels $>16.70 \mathrm{mmol} / \mathrm{L}$ for three measurements were considered diabetic.

Rats proven to be diabetic $(n=50)$ were randomly assigned to five groups with 10 rats in each group: model group, ferulic acid group, astragaloside IV group, ferulic acid + astragaloside IV group, and metformin group. First, the rats were fasted overnight and blood samples were obtained from tails for biochemical determinations including fasting blood glucose (FBG), triglyceride (TG), total cholesterol (TC), low density lipoprotein cholesterol (LDL-C), alanine aminotransferase (ALT), aspartate aminotransferase $(\mathrm{AST})$, creatinine $(\mathrm{Cr})$ and so on. They were administrated with medications as follows: rats were treated with ferulic acid $(50 \mathrm{mg} / \mathrm{kg} \cdot \mathrm{d})$, or astragaloside $\mathrm{IV}(50 \mathrm{mg} / \mathrm{kg} \cdot \mathrm{d})$, or ferulic acid $(50 \mathrm{mg} / \mathrm{kg} \cdot \mathrm{d})+$ astragaloside IV (50 mg/kg•d), or metformin (40.5 mg/ $\mathrm{kg} \cdot \mathrm{d}$ ), respectively by intragastric administration (i.g.) every day for 10 weeks. Rats in the control group and model group were administered with the same volume of normal saline. Body weight of rats in each group was measured every week and tail blood samples were taken to test FBG every two weeks.

All animal experiments were performed in accordance with the guidelines of Shandong University of Traditional Chinese Medicine for the care and use of laboratory animals and approved by the Animal Ethics Review Committee of Shandong University of Traditional Chinese Medicine.

\subsection{Blood and tissue sample collection}

After 10 weeks of drug administration, the rats were fasted overnight, then weighed and anesthetized with $10 \%$ chloralhydrate $(0.3 \mathrm{~g} / \mathrm{kg}$, intraperitoneally). After anesthesia, the rats were fixed, the chest opened to expose the heart, and the aorta was isolated. Blood samples were collected from the apex of the heart, and centrifuged at a speed of 3,000 r/min for $5 \mathrm{~min}$. Serum was removed and aliquots for the respective analytical determinations, was stored at $-80^{\circ} \mathrm{C}$ until analysis. The abdominal aorta was dissected carefully, cleaned of fat and adherent connective tissues. A segment of $15 \mathrm{~mm}$ in length was cut and fixed with $4 \%$ paraformaldehyde for hematoxylin and eosin (HE) staining and immunohistochemical staining, and a segment of $5 \mathrm{~mm}$ in length was cut and stored at $-80^{\circ} \mathrm{C}$ for quantitative real-time RT-PCR and Western blot analysis.

\subsection{Blood samples detection}

According to the manufacture's instructions, the following serum items were detected: First, FPG, TG, TC, LDL-C, ALT, AST and Cr were measured using a fully automatic biochemical analyzer (Olympus, Tokyo, Japan); second, HbAlc was detected to measure long-term blood glucose control; third, concentrations of NO and eNOS in serum were determined by the nitrate reductase method and a colorimetric method respectively.

\subsection{HE and immunohistochemistry staining assay}

To observe the histological morphology changes of the abdominal aorta, HE staining was performed. First, abdominal aorta tissues were fixed with $10 \%$ neutral formaldehyde and dehydrated in graded ethanol $(85 \%$, $95 \%$, and $100 \%$ ). After permeation in xylene, they were embedded in paraffin. The paraffin blocks were cut into $2 \mu \mathrm{m}$ slices, mounted onto glass slides and stained by standard techniques of HE staining according to previous studies. The specimens were examined using a Phenom desktop scanning electron microscope (FEI Company, USA). 
Immunohistochemistry staining assays were performed to analyze the protein expression of TNF- $\alpha$, MCP-1, and NF-кB P65. Paraffin-embedded sections (3-5 $\mu \mathrm{m}$ thick) were cut from formalin-fixed abdominal aortaand carried out as described previously (20). First, paraffin sections were de-paraffinized, hydrated, and immersed in $0.3 \%$ hydrogen peroxide in methanol for 30 min to block the endogenous peroxidase activity. Second, the sections were incubated in primary antibodies (antiTNF- $\alpha$ or anti-MCP-1 or anti-NF- $\kappa$ B P65) overnight at $4^{\circ} \mathrm{C}$, followed by incubation in anti-mouse secondary antibody for $1 \mathrm{~h}$ at room temperature. Third, visualization was carried out using the DAB horseradish peroxidase color development kit, and slides were counter stained in hematoxylin-1. Finally, ten random fields were examined per slice for expression of TNF- $\alpha$, MCP-1, and NF-kB P65 using an Olympus BX40 microscope at a magnification of $\times 400$. Immunohistochemical results of TNF- $\alpha$, MCP-1, and NF- $\kappa$ B P65 expression were assessed according to criteria described previously (21). Five fields for each sample were selected randomly for the calculation. The percentage and staining intensity of the positive cells were observed by Image-Pro plus 6.0 to quantify the expression of proteins: $(i)$ Percentage of positive cells was graded on a scale $0-4$, indicating $0 \%,<$ $10 \%, 10-50 \%, 50-80 \%$, and $80-100 \%$, respectively; (ii) Staining intensity of positive cells was graded on a scale of 0-4, indicating colorless, light yellow, brown yellow, and brown, respectively; (iii) The multiplication of percentage scores and staining intensity scores gave the positive rating: 0 means negative $(-), 1-4$ means weakly positive $(+), 5-8$ means positive $(++)$, and 9-12 means strongly positive $(+++)$.

\subsection{ELISA assay}

ELISA assays were performed to determine the levels of OX-LDL in serum, and TNF- $\alpha$ and MCP-1 in abdominal aorta tissue, using commercially available kits according to the manufacturer's instructions. The optical density was measured on a Bio-Rad Model 680 micro plate reader (Bio-Rad Laboratories, Hercules, CA, USA) at $490 \mathrm{~nm}$.

\subsection{Quantitative real-time RT-PCR assay}

Quantitative real-time RT-PCR was used to detect the mRNA expression of factors related to vascular endothelial dysfunction in the abdominal aorta of diabetic rats. First, total RNA was prepared from the abdominal aorta tissues by using TRIzol reagent according to the manufacturer's instructions. Then, total RNA was reversetranscribed into cDNA using the SuperScript II FirstStrand Synthesis System according to the manufacturer's directions. In the current study, relative mRNA expression levels for the target genes were determined by using glyceraldehyde 3-phosphate dehydrogenase
(GAPDH) as normalization control. The primers for the target genes and GAPDH were shown as follows: TNF- $\alpha$ (198 bp): forward 5'-CTCATTCCTGCTCGTGG-3', and reverse 5'-CTCCGCTTGGTGGTTT-3'; MCP-1 (131 bp): forward 5'-CTGTCACGCTTCTGGG-3' and reverse 5'-GCCGACTCATTGGGAT-3'; GAPDH primers (199 bp): forward 5'-ATCGGACGCCTGGTTA-3' and reverse 5'-CGCTCCTGGAAGATGG-3'. Afterwards, according to the protocol of SYBR Premix Ex TaqTM kit, amplification of target genes and GAPDH were conducted in a $20 \mu \mathrm{L}$ reaction mixture containing $10 \mu \mathrm{L}$ SYBR $(2 \times), 0.2 \mu \mathrm{L}$ ROX Dye II, $2.5 \mu \mathrm{L}$ each forward and reverse primers of each gene, $4 \mu \mathrm{L}$ cDNA and $0.8 \mu \mathrm{L}$ $\mathrm{ddH}_{2} \mathrm{O}$ using ABI Prism 7500 Detection System (Applied Biosystems, Inc., USA). Thermal cycling parameters consisted of a hot-start at $95^{\circ} \mathrm{C}$ for $3 \mathrm{~min}$ followed by 45 cycles of $95^{\circ} \mathrm{C}$ for $20 \mathrm{~s}, 55^{\circ} \mathrm{C}$ for $15 \mathrm{~s}$ and extension at $72^{\circ} \mathrm{C}$ for $20 \mathrm{~s}$. The analysis of relative gene expression was performed by comparative $2^{-\Delta \Delta \mathrm{CT}}$ method (22).

\subsection{Western blot analysis}

Western blot analysis was performed to determine the expression of proteins-related to vascular endothelial dysfunction in the abdominal aorta of diabetic rats. The abdominal aorta was incubated with $50 \mu \mathrm{L}$ RIPA lysis buffer at $4^{\circ} \mathrm{C}$ for $30 \mathrm{~min}$ and then the lysates $(30 \mu \mathrm{g}$ of protein per lane) were fractionated by SDS-PAGE. The proteins were electro-transferred onto polyvinylidene difluoride (PVDF) membranes. The membranes were first incubated in blocking solution (5\% skim milk) for $1 \mathrm{~h}$ at room temperature and then incubated overnight at $4^{\circ} \mathrm{C}$ with the first antibodies: anti-TNF- $\alpha$ or anti-MCP- 1 or anti- NF-кB P65. After they were washed with TBST $(10 \mathrm{mM}$ Tris- $\mathrm{HCl}, 0.15 \mathrm{M} \mathrm{NaCl}, 8 \mathrm{mM}$ sodium azide, $0.05 \%$ Tween-20, $\mathrm{pH} 8.0$ ) three times, the membranes were incubated with horseradish peroxidase-conjugated secondary antibodies for $1 \mathrm{~h}$ and then again washed with TBST three times. Finally, protein bands were visualized with an enhanced chemiluminescence (ECL) detection system. As an internal control, $\beta$-actin was detected with anti- $\beta$-actin antibodies.

\subsection{Statistical analysis}

All experiments were performed in triplicate and the results are expressed as mean \pm S.D. Statistical analysis was performed with one-way analysis of variance (ANOVA) and the Student's $t$-test using SPSS.15.0 software (SPSS Inc., USA). $P<0.05$ was indicative of a significant difference.

\section{Results}

\subsection{The general condition of rats}

After treatment for 10 weeks, the rats in the control 
Table 1. The effects of ferulic acid and astragaloside IV on biochemical indexes and HbAlc of rats

\begin{tabular}{|c|c|c|c|c|c|}
\hline Group & FPG (mmol/L) & HbAlc (\%) & $\mathrm{TG}(\mathrm{mmol} / \mathrm{L})$ & $\mathrm{TC}(\mathrm{mmol} / \mathrm{L})$ & LDL-C $(\mathrm{mmol} / \mathrm{L})$ \\
\hline Control & $5.43 \pm 0.23$ & $5.4 \pm 0.2$ & $0.85 \pm 0.13$ & $1.88 \pm 0.10$ & $0.30 \pm 0.06$ \\
\hline Model & $22.28 \pm 2.09$ & $14.5 \pm 0.6$ & $2.01 \pm 0.09$ & $2.79 \pm 0.12$ & $5.20 \pm 0.12$ \\
\hline Ferulic acid & $21.64 \pm 1.25$ & $14.6 \pm 0.5$ & $1.11 \pm 0.18^{* *}$ & $2.10 \pm 0.19^{* *}$ & $0.32 \pm 0.10^{* *}$ \\
\hline Astragaloside IV & $12.92 \pm 2.94^{*}$ & $9.5 \pm 0.4^{* *}$ & $1.94 \pm 0.11$ & $2.64 \pm 0.16$ & $0.65 \pm 0.07$ \\
\hline Ferulic acid + Astragaloside IV & $12.72 \pm 2.84^{* *}$ & $9.2 \pm 0.2^{* *}$ & $1.08 \pm 0.16^{* *}$ & $2.38 \pm 0.10^{* *}$ & $0.38 \pm 0.06^{* *}$ \\
\hline Metformin & $7.64 \pm 1.06^{* *}$ & $8.9 \pm 0.4^{* *}$ & $0.85 \pm 0.13$ & $1.88 \pm 0.10$ & $0.50 \pm 0.20$ \\
\hline
\end{tabular}

Data are shown as mean \pm S.D. $(n=10) .{ }^{*} p<0.05$ and ${ }^{* *} p<0.01$ versus Model group after treatment for 10 weeks.

group were in good shape, glossy coat color and obesity, while the rats in the model group and drug treatment groups had different degrees of low spirits, matte coat color, and typical characteristics of diabetes mellitus (polydipsia, polyphagia, polyuria, and weakness). In addition, there was a great change in body weight of rats after treatment with ferulic acid and astragaloside IV (Data not shown). After 10 weeks of treatment, body weights in each group increased with the control group increasing the most and the model group increasing the minimum $(p<0.01)$. Body weights in metformin group $(p<0.01)$, astragaloside IV group $(p<0.05)$ and ferulic acid + astragaloside IV group $(p<0.05)$ were higher compared to the model group. However, there was no significant difference between body weights in the ferulic acid group compared to the model group ( $p$ $>0.05$ ).

\subsection{The effects of ferulic acid and astragaloside IV on} biochemical indexes and HbAlc of rats

The effects of ferulic acid and astragaloside IV on biochemical indexes (e.g., FPG, TG, TC, and LDL-C) of diabetic rats are presented in Table 1. Compared to model group, levels of FPG in metformin group ( $p<$ $0.01)$, astragaloside IV group $(p<0.05)$ and ferulic acid + astragaloside IV group $(p<0.05)$ decreased significantly. However, there was no significant difference between FPG in the ferulic acid group and the model group $(p>0.05)$. Compared to the model group, the levels of HbAlc, TG, TC and LDL-C decreased significantly in the ferulic acid + astragaloside IV group $(p<0.01)$. Compared to the model group, both in the metformin group and astragaloside IV group, levels of HbAlc decreased significantly $(p<0.01)$ but the levels of TG, TC and LDL-C decreased non-significantly ( $p$ $>0.05)$. Compared to the model group, in the ferulic acid group, levels of TG, TC and LDL-C decreased significantly $(p<0.01)$ but levels of HbAlc decreased non-significantly $(p>0.05)$. Taken together, ferulic acid combined with astragaloside IV exhibited a significant effect on decreasing the levels of FPG, HbAlc, TG, $\mathrm{TC}$, and LDL-C. In addition, there was no significant difference between ALT, AST, and Cr before and after treatment with ferulic acid and astragaloside IV (data not shown).

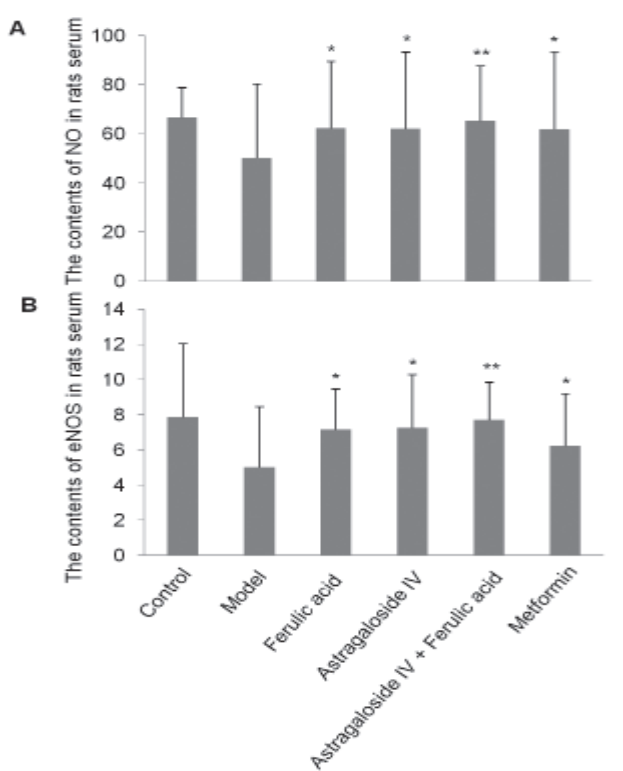

Figure 2. The contents of NO (A) and NOS (B) in the serum of diabetic rats. The data represent mean \pm S.D. $(n=$ 10). ${ }^{*} p<0.05$ and ${ }^{* *} p<0.01$ versus Model group.

\subsection{The effects of ferulic acid and astragaloside IV on $N O$ and eNOS of rats}

After treatment for 10 weeks, the amount of $\mathrm{NO}$ and eNOS in serum were detected. As shown in Figure 2, the amount of NO and eNOS decreased in the model group and drug treatment groups compared to the control group. The amount of NO and eNOS in each drug treatment group $(p<0.05)$ especially ferulic acid + astragaloside IV group $(p<0.01)$ were higher than those in the model group. That is to say, compared to the model group, the amount of NO and eNOS in each drug treatment group increased. Moreover, in the ferulic acid + astragaloside IV group, the amount of NO and eNOS increased significantly compared to other treatment groups $(p<0.01)$.

\subsection{The effects of ferulic acid and astragaloside $I V$ on the histological morphology changes of abdominal aorta of rats}

As shown in Figure $3 \mathrm{~A}$, in the control group, the 


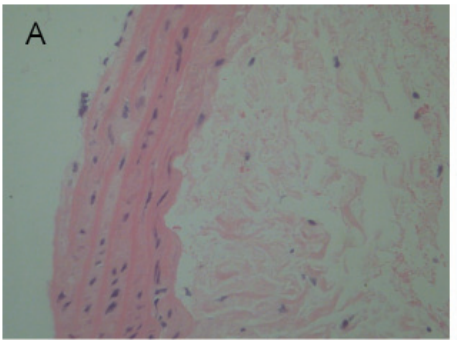

Control

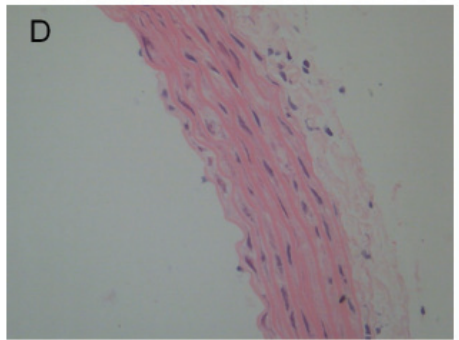

Astragaloside IV

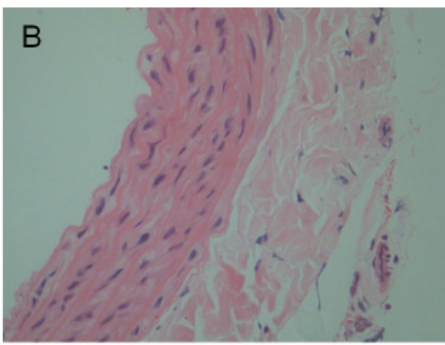

Model

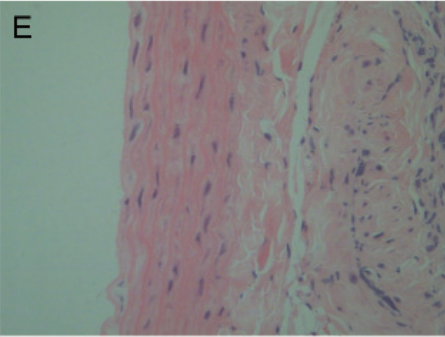

Astragaloside IV + Ferulic acid

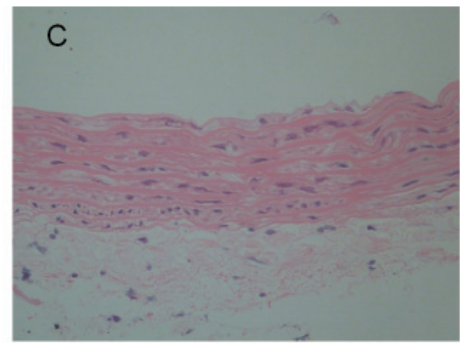

Ferulic acid

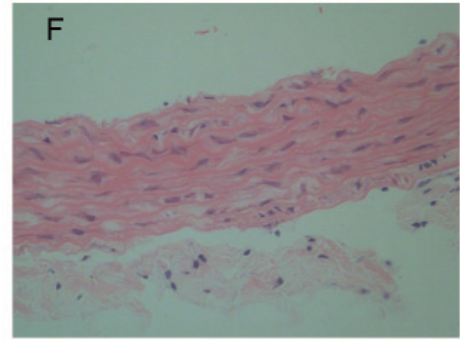

Metformin

Figure 3. The histological morphology changes of abdominal aorta endothelium of diabetic rats in each group (original magnification ×400). (A) Control group; (B) Model group; (C) Ferulic acid group; (D) Astragaloside IV group; (E) Ferulic acid + Astragaloside IV group; (F) Metformin group.

abdominal aorta intima was smooth, endothelial cell integrity was preserved, the single layer was close to the elastic plate and medial thickness was uniform. However, in the model group (Figure 3B), the typical three-layer structure of the aortic wall was not clear, and intimal thickness increased with irregular intima. In the drug treatment groups especially in ferulic acid combined with astragaloside IV group (Figure 3C-3F), the aortic intima was smooth and intimal thickening was less compared with the model group. Progression of aorta intima and endothelial structure were attenuated by ferulic acid and astragaloside IV therapy. In the ferulic acid + astragaloside IV group, as shown in Figure 3E, intima was smooth with little endothelial cell drop, and media smooth muscle cells proliferated with uniform thickness and regular arrangement.

\subsection{The effects of ferulic acid and astragaloside $I V$ on} the expression of $O x-L D L, M C P-1, T N F-\alpha$ and $N F-\kappa B$ P65

The expression of Ox-LDL in serum and the expression of MCP-1 and TNF- $\alpha$ in the abdominal aorta tissue were detected by ELISA assays. As shown in Figure 4, compared to the control group, both the expression of Ox-LDL, MCP-1, and TNF- $\alpha$ in the model group and each drug treatment group increased significantly. Furthermore, compared to the model group, the expression of Ox-LDL were significantly inhibited after treatment with ferulic acid single $(p<0.01)$ or ferulic acid combined with astragaloside IV $(p<0.01)$. Compared to the model group, the expression of MCP1 and TNF- $\alpha$ were significantly inhibited after treatment with ferulic acid and astragaloside IV especially in

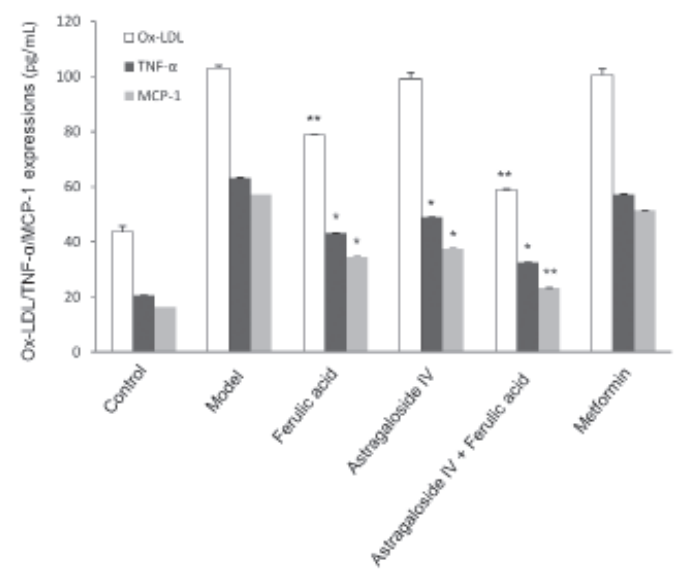

Figure 4. The expressions of Ox-LDL in serum of rats and the expressions of MCP-1 and TNF- $\alpha$ in the abdominal aorta tissue of rats detected by ELISA assay. The data represent mean \pm S.D. $(n=10) . * p<0.05$ and $* * p<0.01$ versus Model group.

the ferulic acid + astragaloside IV group $(p<0.01)$. However, the expression of Ox-LDL, MCP-1, and TNF- $\alpha$ was inhibited insignificantly in the metformin group compared to the model group. Taken together, ferulic acid combined with astragaloside IV exhibited the greatest effect on inhibiting the expression of OxLDL, MCP-1, and TNF- $\alpha$ compared to only treatment with ferulic acid, astragaloside IV or metformin alone.

The mRNA expressionof MCP-1 and TNF- $\alpha$ in the abdominal aorta of diabetic rats were detected by quantitative real-time RT-PCR assays. As shown in Figure 5, compared to the control group, the mRNA expression of MCP-1 and TNF- $\alpha$ in the model group and each drug treatment group increased significantly. Furthermore, compared to the model group, the 


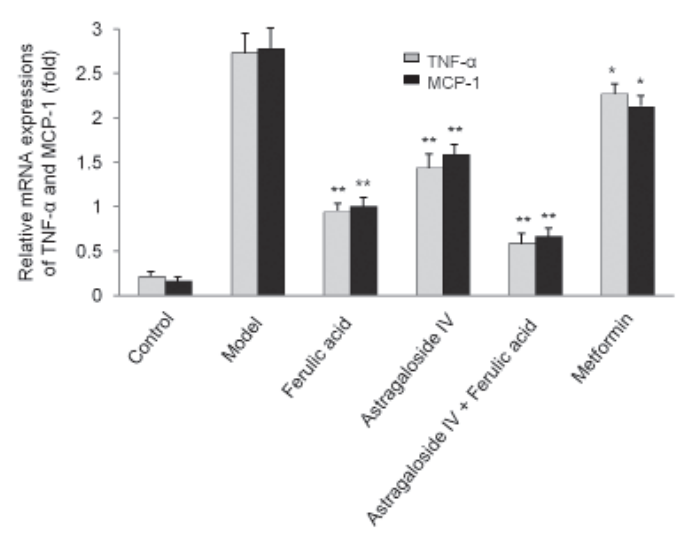

Figure 5. The mRNA expressions of MCP-1 and TNF- $\alpha$ in the abdominal aorta of diabetic rats detected by quantitative real-time RT-PCR assay. The data represent mean \pm S.D. $(n=10) .{ }^{*} p<0.05$ and ${ }^{* *} p<0.01$ versus Model group.

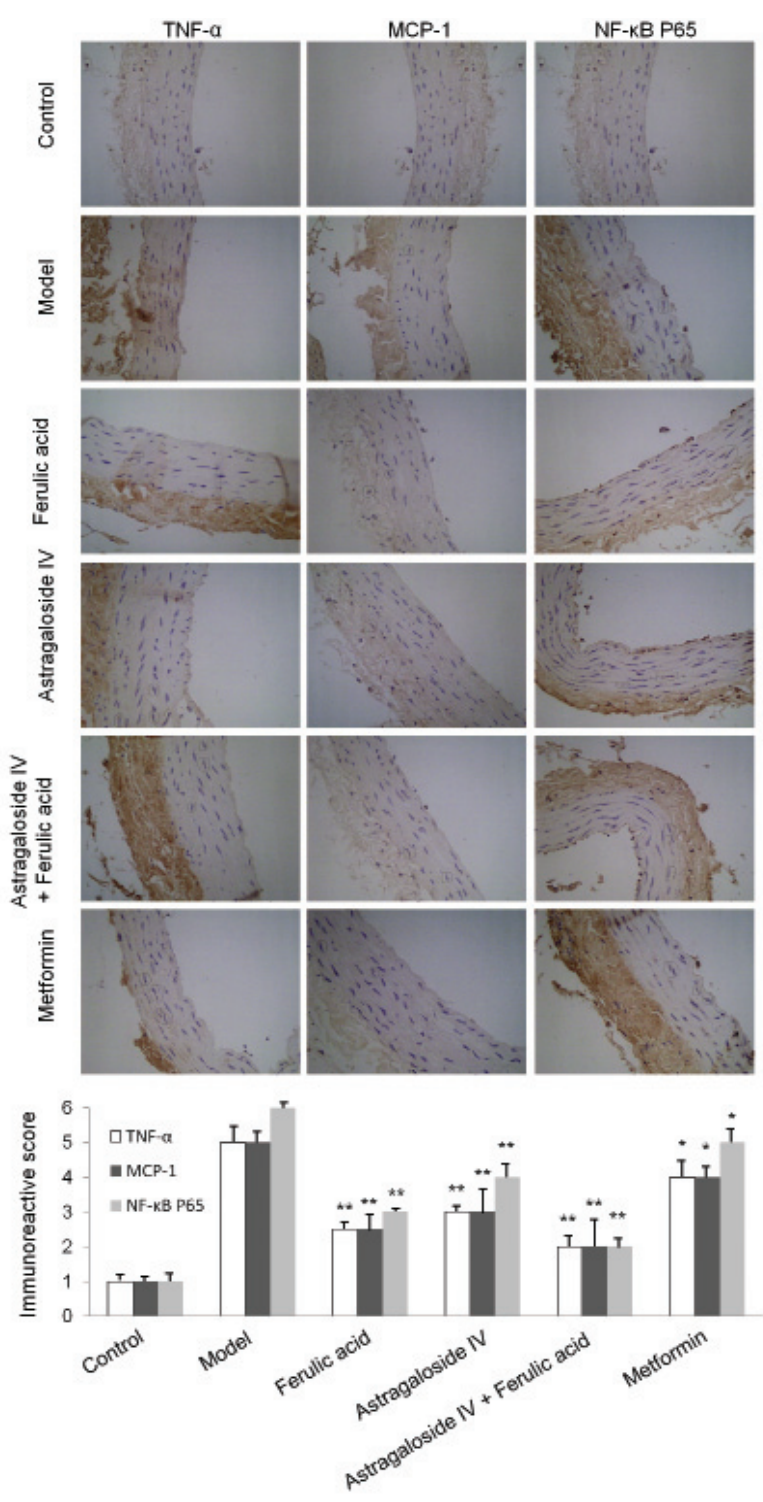

Figure 6. The protein expression of TNF- $\alpha$, MCP-1 and NF-кB P65 in the abdominal aorta of diabetic rats in each group detected by immunohistochemistry staining assay (original magnification $\times 400$ ).

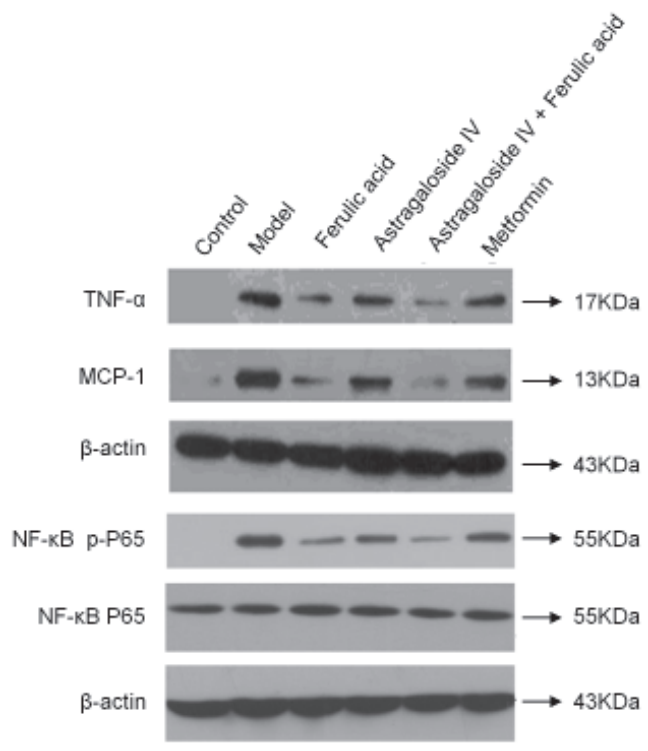

Figure 7. The protein expression of TNF- $\alpha$, MCP-1, and NF- $\kappa$ B P65 in the abdominal aorta of diabetic rats in each group detected by Western blot analysis.

expression of MCP-1 and TNF- $\alpha$ were significantly inhibited in each drug treatment group especially in the ferulic acid + astragaloside IV group $(p<0.01)$. Ferulic acid combined with astragaloside IV exhibited the greatest effect on inhibiting the mRNA expression of MCP- 1 and TNF- $\alpha$ compared to only treatment with ferulic acid, astragaloside IV or metformin alone.

The protein expression of TNF- $\alpha, \mathrm{MCP}-1$, and NF- $\mathrm{B}$ P65 were detected by immunohistochemistry staining and Western blot analysis. As shown in Figure 6 and Figure 7, compared to the control group, the expression of TNF- $\alpha, \mathrm{MCP}-1$, and NF- $\kappa \mathrm{B}$ P65 increased significantly. Furthermore, compared to the model group, the levels of TNF- $\alpha$, MCP-1, and NF$\kappa$ B P65 were significantly down-regulated in each drug treatment group especially in the ferulic acid + astragaloside IV group. Ferulic acid combined with astragaloside IV exhibited the greatest effect on inhibiting the mRNA expression of MCP-1 and TNF- $\alpha$ compared to other treatment.

\section{Discussion}

Dysfunction of the endothelium is regarded as an important factor in the pathogenesis of vascular disease in DM. In this work, we evaluated the protective effect of ferulic acid and astragaloside IV against vascular endothelial dysfunction in streptozotocininduced diabetic rats. The major finding of this study was that ferulic acid combined with astragaloside IV was capable of improving the structure of the aortic endothelium wall (Figure 3), attenuating the increase of FPG, HbAlc, TG, TC, LDL-C (Table 1) and Ox-LDL (Figure 4), promoting the release of NO (Figure 2), and inhibiting over-activation of MCP-1, TNF- $\alpha$, and NF- 
кB P65 (Figures 4-7). Moreover, there was no damage to liver and kidney function after treatment with ferulic acid and astragaloside IV for 10 weeks. In a word, our data contribute in part to elucidate the molecular mechanisms involved in the protective effect of ferulic acid and astragaloside IV against vascular endothelial dysfunction in DM with little side effects. Ferulic acid combined with astragaloside IV might exhibit a significant protective effect against vascular endothelial dysfunction in DM via inhibiting the NF- $\kappa \mathrm{B}$ pathway involving a decrease of Ox-LDL, an increase of NO and eNOS, and activation of NF- $\mathrm{B}$ P65, MCP-1, and TNF- $\alpha$.

Hyperglycemia is generally considered as a harmful factor in vascular dysfunction, and the focus has been on tight blood glucose control as a treatment for diabetic patients (3). In the current study, we found that ferulic acid combined with astragaloside IV could effectively reduce the levels of FPG and HbAlc. Dyslipidemia is a major risk factor for atherosclerosis. Reducing high levels of TC, particularly LDL-C, decreases the risk for developing atherosclerosis (23). We found that ferulic acid combined with astragaloside IV could effectively reduce the levels of TG, TC, and LDL-C, and decrease the risk for vascular dysfunction in DM.

DM has been known as an oxidative stress disorder which is caused by imbalance between free radical formation and the ability of the body's natural antioxidants in hyperglycemia (24). Many studies have suggested that oxidative stress plays a key role in the pathogenesis of endothelial dysfunction, insulin resistance, and pancreatic $\beta$ cell damage and so on in development and progression of DM as well as in complications (25). As a major product of oxidative stress, Ox-LDL induces apoptosis, monocyte adhesion, and reactive oxygen species generation via upregulating and binding to its receptor LOX-1, and then causes toxicity to the vascular endothelial cells and causes endothelial dysfunction, which is considered to be a driving force in the initiation and development of atherosclerosis. Notably, the toxicity of Ox-LDL is not only damage of vascular endothelial structure, but more important is that Ox-LDL affects the secretion of endothelium-derived vasomotor factors and adhesion factors which leads to endothelial dysfunction (23). NO is an important vasomotor factor and endothelin (ET) is a stronger vasoconstrictor factor, which are secreted by vascular endothelial cells. Keeping balance between the levels of NO and ET is important in the maintenance of vascular endothelium diastolic function (26). Moreover, the production of NO in endothelial cells is mainly regulated by eNOS which is a nitric oxide synthase that generates NO in blood vessels and is involved with regulating vascular tone by inhibiting smooth muscle contraction and platelet aggregation (27). However, the production or activation of eNOS and NO could be reduced in endothelial cells attributed to oxidative stress and the increase of Ox-LDL in DM patients. Thus, regulating the activity of eNOS and promoting NO production might be key targets for prevention of endothelial dysfunction in DM. In the current study, we found that the levels of OxLDL were significantly decreased by ferulic acid and astragaloside IV (Figure 4). Furthermore, the activity of eNOS and the amount of NO in serum of diabetic rats were effectively improved after treatment with ferulic acid and astragaloside IV (Figure 2). That is to say, ferulic acid combined with astragaloside IV exhibited a significant protective effect against vascular endothelial dysfunction in DM via decreasing Ox-LDL and increasing NO.

Some studies demonstrated that enhanced NF$\kappa \mathrm{B}$ activity is an important factor involved in macroand microvascular dysfunction in diabetes. It is closely related to DM-associated oxidative stress and inflammation (28). In the state of hyperglycemiainduced oxidative stress, the elevation of free radicals could impair $\mathrm{Ca}^{2+}$ translocation and then trigger NF$\kappa \mathrm{B}$ activation, reducing vascular contractibility and modifying some gene expression including cytokines (e.g., IL-6, TNF- $\alpha$ ), angiotensin-II and adhesion molecules (e.g., ICAM-1, VCAM-1, and E-selectin) and chemokines (e.g., MCP-1, IL-8) released from vascular endothelial cells and causing endothelial dysfunction (29). Importantly, as well as hyperglycemia, cytokine stimulation (e.g., TNF- $\alpha$, IL-1 $\beta$, MCP-1, and ICAM1) also could activate $\mathrm{NF}-\kappa \mathrm{B}$ via reactive oxygen species formation. In addition, $\mathrm{NF}-\kappa \mathrm{B}$ is one of the most important regulators of pro-inflammatory gene expression. In DM models, activation of NF- $\mathrm{DB}$ is correlated with an increased of some cytokine proinflammatory molecules. NF- $\kappa \mathrm{B}$ could lead to or enhance expression of host genes, such as cytokine TNF- $\alpha$ and chemokine MCP-1, inducing inflammatory damage of vascular wall, and then initiating atherosclerosis (30). Thus, NF- $\kappa \mathrm{B}$ plays critical roles in the pathophysiology of several process of vascular endothelial dysfunction in DM, and it is plausible that exogenous modulation of $\mathrm{NF}-\kappa \mathrm{B}$ activation may be effective in developing new therapeutic strategies. In the current study, we found that gene and protein expression of MCP-1, TNF- $\alpha$, and NF- $\kappa$ B P65 were significantly inhibited by ferulic acid and astragaloside IV (Figures 4-7). That is to say, ferulic acid combined with astragaloside IV exhibited a significant protective effect against vascular endothelial dysfunction in DM via inhibiting the NF- $\mathrm{B}$ pathway.

In conclusion, the current study demonstrated that ferulic acid combined with astragaloside IV could effectively reduce the levels of FPG, HbAlc, TG, TC, and LDL-C. The current study also demonstrated that ferulic acid combined with astragaloside IV exhibited a significant protective effect against vascular endothelial dysfunction in diabetic rats through the NF- $\mathrm{B}$ pathway 
involving decrease of Ox-LDL, an increase of NO and eNOS, and activation of NF- $\kappa \mathrm{B}$ P65, MCP-1, and TNF- $\alpha$. We hope the current study provides a direction of new therapy for treating vascular complications of DM.

\section{Acknowledgements}

This project was supported by the science and technology development plan of traditional Chinese medicine in Shandong Province, China (No. 2013057).

\section{References}

1. Shaw JE, Sicree RA, Zimmet PZ. Global estimates of the prevalence of diabetes for 2010 and 2030. Diabetes Res Clin Pract. 2010; 87:4-14.

2. Saxena B. Anti-hyperlipidemic activity of Withania coagulans in streptozotocininduced diabetes: A potent anti-atherosclerotic agent. Drug Discov Ther. 2010; 4:334-340.

3. Goff DC Jr, Gerstein HC, Ginsberg HN, Cushman WC, Margolis KL, Byington RP, Buse JB, Genuth S, Probstfield JL, Simons-Morton DG; ACCORD Study Group. Prevention of cardiovascular disease in persons with type 2 diabetes mellitus: Current knowledge and rationale for the Action to Control Cardiovascular Risk in Diabetes (ACCORD) trial. Am J Cardiol. 2007; 99:4i-20i.

4. Beckman JA, Creager MA, Libby P. Diabetes and atherosclerosis: epidemiology, pathophysiology, and management. JAMA. 2002; 287:2570-2581.

5. Capellini VK, Celotto AC, Baldo CF, Olivon VC, Viaro F, Rodrigues AJ, Evora PR. Diabetes and vascular disease: Basic concepts of nitric oxide physiology, endothelial dysfunction, oxidative stress and therapeutic possibilities. Curr Vasc Pharmacol. 2010; 8:526-544.

6. Tabak O, Gelisgen R, Erman H, Erdenen F, Muderrisoglu C, Aral H, Uzun H. Oxidative lipid, protein, and DNA damage as oxidative stress markers in vascular complications of diabetes mellitus. Clin Invest Med. 2011; 34:E163-E171.

7. Tousoulis D, Papageorgiou N, Androulakis E, Siasos G, Latsios G, Tentolouris K, Stefanadis C. Diabetes mellitus-associated vascular impairment: Novel circulating biomarkers and therapeutic approaches. J Am Coll Cardiol. 2013; 62:667-676.

8. Rochette L, Lorin J, Zeller M, Guilland JC, Lorgis L, Cottin Y, Vergely C. Nitric oxide synthase inhibition and oxidative stress in cardiovascular diseases: Possible therapeutic targets? Pharmacol Ther. 2013; 140:239-257.

9. Polovina MM, Potpara TS. Endothelial dysfunction in metabolic and vascular disorders. Postgrad Med. 2014; 126:38-53.

10. Tzeng TF, Liou SS, Liu IM. The selected traditional Chinese medicinal formulas for treating diabetic nephropathy: perspective of modern science. J Tradit Complement Med. 2013; 3:152-158.

11. Xie W, Du L. Diabetes is an inflammatory disease: Evidence from traditional Chinese medicines. Diabetes Obes Metab. 2011; 13:289-301.

12. Zhang WL, Zheng KY, Zhu KY, Zhan JY, Bi CW, Chen JP, Dong TT, Choi RC, Lau DT, Tsim KW. Chemical and biological assessment of angelica roots from different cultivated regions in a Chinese herbal decoction danggui buxue tang. Evid Based Complement Alternat Med. 2013; 2013:483286.

13. Chao WW, Lin BF. Bioactivities of major constituents isolated from Angelica sinensis (Danggui). Chin Med. 2011; 6:29.

14. Lekakis J, Rallidis LS, Andreadou I, Vamvakou G, Kazantzoglou G, Magiatis P, Skaltsounis AL, Kremastinos DT. Polyphenolic compounds from red grapes acutely improve endothelial function in patients with coronary heart disease. Eur J Cardiovasc Prev Rehabil. 2005; 12:596-600.

15. Yuan W, Zhang Y, Ge Y, Yan M, Kuang R, Zheng X. Astragaloside IV inhibits proliferation and promotes apoptosis in rat vascular smooth muscle cells under high glucose concentration in vitro. Planta Med. 2008; 74:1259-1264.

16. Hoo RL, Wong JY, Qiao C, Xu A, Xu H, Lam KS. The effective fraction isolated from Radix Astragali alleviates glucose intolerance, insulin resistance and hypertriglyceridemia in $\mathrm{db} / \mathrm{db}$ diabetic mice through its anti-inflammatory activity. Nutr Metab (Lond). 2010; $7: 67$.

17. Ren S, Zhang H, Mu Y, Sun M, Liu P. Pharmacological effects of astragaloside IV: A literature review. J Tradit Chin Med. 2013; 33:413-416.

18. Gao D, Guo Y, Li X, Li X, Li Z, Xue M, Ou Z, Liu M, Yang M, Liu S, Yang S. An aqueous extract of Radix astragali, Angelica sinensis, and Panax notoginseng is effective in preventing diabetic retinopathy. Evid Based Complement Alternat Med. 2013; 2013:578165.

19. Liu IM, Tzeng TF, Liou SS. A Chinese herbal decoction, Dang Gui Bu Xue Tang, prepared from Radix astragali and Radix angelicae sinensis, ameliorates insulin resistance induced by A high-fructose diet in Rats. Evid Based Complement Alternat Med. 2011; 2011:248231.

20. Hu L, Xue F, Shao MH, Deng AM, Wei GT. Aberrant expression of Notch3 predicts poor survival for hepatocellular carcinomas. Biosci Trends. 2013; 7:152156.

21. Soslow RA, Dannenberg AJ, Rush D, Woerner BM, Khan KN, Masferrer J, Koki AT. Cox-2 is expressed in human pulmonary, colonic, and mammary tumors. Cancer. 2000; 89:2637-2645.

22. Livak KJ, Schmittgen TD. Analysis of relative gene expression data using real-time quantitative PCR and the $2^{-\Delta \Delta C_{T}}$ method. Methods. 2001; 25:402-408.

23. Kita T, Kume N, Minami M, Hayashida K, Murayama T, Sano H, Moriwaki H, Kataoka H, Nishi E, Horiuchi H, Arai H, Yokode M. Role of oxidized LDL in atherosclerosis. Ann N Y Acad Sci. 2001; 947:199-206.

24. Zatalia SR, Sanusi H. The role of antioxidants in the pathophysiology, complications, and management of diabetes mellitus. Acta Med Indones. 2013; 45:141-147.

25. Pitocco D, Tesauro M, Alessandro R, Ghirlanda G, Cardillo C. Oxidative stress in diabetes: Implications for vascular and other complications. Int J Mol Sci. 2013; 14:21525-21550.

26. Pitocco D, Tesauro M, Alessandro R, Ghirlanda G, Cardillo C. Oxidative stress in diabetes: Implications for vascular and other complications. Int J Mol Sci. 2013; 14:21525-21550.

27. Lu YL, Hu SJ, Shen ZJ, Shao YC. Changes of macrovascular endothelial ultrastructure and gene 
expression of endothelial nitric oxide synthase in diabetic rats.Chin Med J (Engl). 2004; 117:1165-1169.

28. Lorenzo O, Picatoste B, Ares-Carrasco S, Ramírez E, Egido J, Tuñón J. Potential role of nuclear factor $\kappa \mathrm{B}$ in diabetic cardiomyopathy. Mediators Inflamm. 2011; 2011:652097.

29. de Winther MP, Kanters E, Kraal G, Hofker MH. Nuclear factor kappaB signaling in atherogenesis. Arterioscler
Thromb Vasc Biol. 2005; 25:904-914.

30. Palomer X, Salvadó L, Barroso E, Vázquez-Carrera M. An overview of the crosstalk between inflammatory processes and metabolic dysregulation during diabetic cardiomyopathy. Int J Cardiol. 2013; 168:3160-3172.

(Received July 22, 2014; Revised August 4, 2014; Accepted August 5, 2014) 